\title{
Eficacia a largo plazo del tratamiento endoscópico en el reflujo vesicoureteral de etiología secundaria con polidimetilsiloxano
}

\author{
Serrano Durbá A, Gómez Pérez L, Estornell Moragues JF, Domínguez Hinarejos C, \\ Martínez Verduch M, García Ibarra F.
}

Servicio de Urología Infantil. Hospital Universitario La Fe. Valencia

Actas Urol Esp. 2006;30(7):692-697

\section{RESUMEN}

\section{EFICACIA DEL TRATAMIENTO ENDOSCÓPICO EN EL REFLUJO VÉSICOURETERAL DE ETIOLOGÍA} SECUNDARIA CON POLIDIMETILSILOXANO

Objetivo: Valorar la eficacia del tratamiento endoscópico del reflujo vesicoureteral (RVU) de etiología secundaria con polidimetilsiloxano.

Material y método: Hemos realizado un estudio prospectivo de los pacientes diagnosticados de RVU y tratados en nuestro servicio por vía endoscópica con polidimetilsiloxano, desde enero de 1999 a diciembre de 2001, analizando los resultados tras un seguimiento medio de 30 meses. Tratamos 144 pacientes, 92 niñas y 52 niños, con 213 reflujos ureterales (124 primarios y 89 de etiologia secundaria). La eficacia inicial del tratamiento fue definida como la ausencia completa de RVU en la cistografía isotópica realizada un los tres meses tras la punción.

Resultados: La eficacia del global tratamiento endoscópico en reflujos de etiología secundaria fue inferior al alcanzado en el tratamiento del RVU primario (el 77,7 \% vs. 86,2 \%) sin alcanzar estas diferencias la significación estadística $(\mathrm{p}=0,226)$. Tampoco encontramos diferencias significativas al comparar el volumen inyectado y la tasa de resolución del reflujo entre las diferentes etiologías de RVU ( $\mathrm{p}=0,361)$. Únicamente se objetivó recurrencia del RVU en pacientes con reflujo secundario a disfunción neurogénica o por disfunción de vaciado $(4,34 \%)$.

Conclusiones: El tratamiento endoscópico del RVU de etiología secundaria es un procedimiento mínimamente invasivo, presenta escasa morbilidad y es eficaz en el RVU de etiología secundaria en pacientes seleccionados. El riesgo de recurrencia a largo plazo es mayor en el reflujo secundario a alteraciones funcionales (vejiga neurógena e inestabilidad funcional), por lo que el seguimiento debería establecerse según la patología de base.

Palabras clave: Reflujo vesicoureteral. Tratamiento endoscópico. Pediatría.

\section{ABSTRACT \\ EFFICACY IN ENDOSCOPIC TREATMENT OF SECONDARY VESICOURETERAL REFLUX WITH POLYDIMETHYLSILOXANE}

Objective: To value the efficacy in endoscopic treatment of the vesicoureteral reflux (VUR).

Material and method: We have realized a prospective study in patients with RVU treated with endoscopic polydimethylsiloxane, from January 1999 until December 2001, analyzing the results after an average pursuit of 30 months. We included 144 patients, 92 girls and 52 children, with 213 VUR (124 primary ones and 89 of secondary etiology). The initial efficacy of the treatment was defined as the finished absence of RVU in the isotopic cystography, realized three months after the puncture.

Results: The global efficacy of the endoscopic treatment in secondary etiology reflux was lower than the reached one in the treatment of the primary RVU (77.7\% against $86.2 \%)$ but the differences did not reach statistical significance $(\mathrm{p}=0,226)$. We do not also find significant differences on having compared the injected volume and the valuation of resolution of the ebb between the different causes of secondary RVU ( $\mathrm{p}=0.361$ ). We found recurrence in patients with RVU due to lower urinary tract dysfunction (4.34\%).

Conclusions: Endoscopic treatment of the secondary VUR is a minimally invasive skill, presents scarce morbidity and it is effective in chosen patients. The risk of a long term recurrence is grater in VUR secondary to functional alterations (neurogenic bladder and functional instability), for what, pursuit has to be established according to the base pathology. 
$\mathrm{E}^{1}$ reflujo vesicoureteral (RVU) es la patología más frecuente en urología pediátrica, con una prevalencia del $1 \%$ en la población infantil, llegando hasta el 25-49\% en niños con infección del tracto urinario superior ${ }^{1}$. La posible repercusión de las infecciones sobre las unidades renales, tales como insuficiencia renal e hipertensión arterial, obliga al seguimiento y la corrección del RVU en algunos pacientes ${ }^{2}$.

El tratamiento del RVU por vía endoscópica fue descrito por Matouscheck en $1981^{3}$ y posteriormente desarrollado por O’Donnell y Puri ${ }^{4}$. Actualmente la inyección endoscópica de agentes inertes en el RVU primario ha demostrado ser un procedimiento eficaz, poco invasivo, con escasa morbilidad y estancia hospitalaria reducida ${ }^{5-8}$.

Respecto al tratamiento del RVU de etiología secundaria, también se ha utilizado con éxito el abordaje endoscópico en algunos casos de reflujo asociado a disfunción miccional neurógena y no neurógena, sistemas dobles y ureterocele, fracaso del reimplante quirúrgico y secundario a patología obstructiva del tracto inferior ${ }^{9-11}$. Sin embargo la experiencia es limitada. Falta todavía precisar cuándo estaría realmente indicado el tratamiento endoscópico (TE) en reflujos secundarios, en qué pacientes, cuales son sus complicaciones en estas indicaciones, si su eficacia es mantenida en el tiempo y cómo debería realizarse el seguimiento una vez realizado el tratamiento.

El objetivo de este trabajo es valorar nuestros resultados a largo plazo en el tratamiento endoscópico del RVU secundario.

\section{MATERIAL Y MÉTODO}

Hemos realizado un estudio prospectivo de los pacientes diagnosticados de RVU y tratados en nuestro servicio por vía endoscópica desde enero de 1999 a diciembre de 2001, analizando los resultados tras un seguimiento medio de 30 meses (15 a 36 meses).

Tratamos 144 pacientes, 92 niñas y 52 niños, con 213 reflujos ureterales. La edad de los pacientes estaba com-

Tabla 1 prendida entre 1 y 16 años, con una media de 7,18 años.

Los pacientes tratados fueron divididos en dos grupos según la etiología del reflujo:

- grupo I, 124 unidades ureterales con RVU primario $(58,21 \%)$ y

- grupo II, constituido por 89 unidades ureterales con RVU secundario, que representaban el 41,79\% del total.

La etiología y grado del reflujo secundario se refleja en Tabla 1 y la Figura 2.

Los criterios para el tratamiento fueron la existencia de reflujo de alto grado, infecciones de repetición pese a tratamiento conservador, deterioro de la función renal y edad superior al año de vida. En RVU por válvulas de uretra posterior o estenosis uretral, la indicación para tratamiento endoscópico se estableció en función del estudio urodinámico, tras tratamiento de la patología de base (derivación urinaria o resección de válvulas, uretroplastia o uretrotomía). En pacientes con RVU persistente tras reimplante, fueron tratados el reflujo sintomático en ambos sexos y el asintomático en niñas antes de entrar en la pubertad. En pacientes con reflujo secundario a alteración funcional del tracto inferior, seleccionamos para tratamiento los RVU persistentes a pesar de un adecuado control de la patología de base y los pacientes con infecciones de repetición.

El material utilizado para la inyección endoscópica fue el polidimetilsiloxano en todos los pacientes, aplicado según la técnica de inyección clásica descrita por Puri ${ }^{12}$. Todos los procedimientos fueron cirugias sin ingreso hospitalario.

Grado de reflujo según etiología

\begin{tabular}{lcccc}
\hline \multicolumn{1}{l}{ Grado de reflujo vésico ureteral } & I & II & III & IV \\
\hline Alteración funcional* & 2 & 17 & 13 & 0 \\
Duplicidad ureteral & 1 & 10 & 9 & 5 \\
Vejiga neurógena & $1(6,7 \%)$ & $5(33,3 \%)$ & $7(46,7 \%)$ & $2(13,3 \%)$ \\
Reimplante ureteral & 0 & $2(28,6 \%)$ & $3(42,9 \%)$ & $2(28,6 \%)$ \\
Ureterocele puncionado & $2(33,3 \%)$ & $2(33,3 \%)$ & $2(33,3 \%)$ & $1(14,3 \%)$ \\
Obstrucción orgánica** & 0 & $1(33,3 \%)$ & $1(33,3 \%)$ & $1(33,3 \%)$ \\
\hline
\end{tabular}

*Incoordinación vesicoesfinteriana o hiperactividad vesical.

**Estenosis de uretra y válvulas de uretra posterior. 


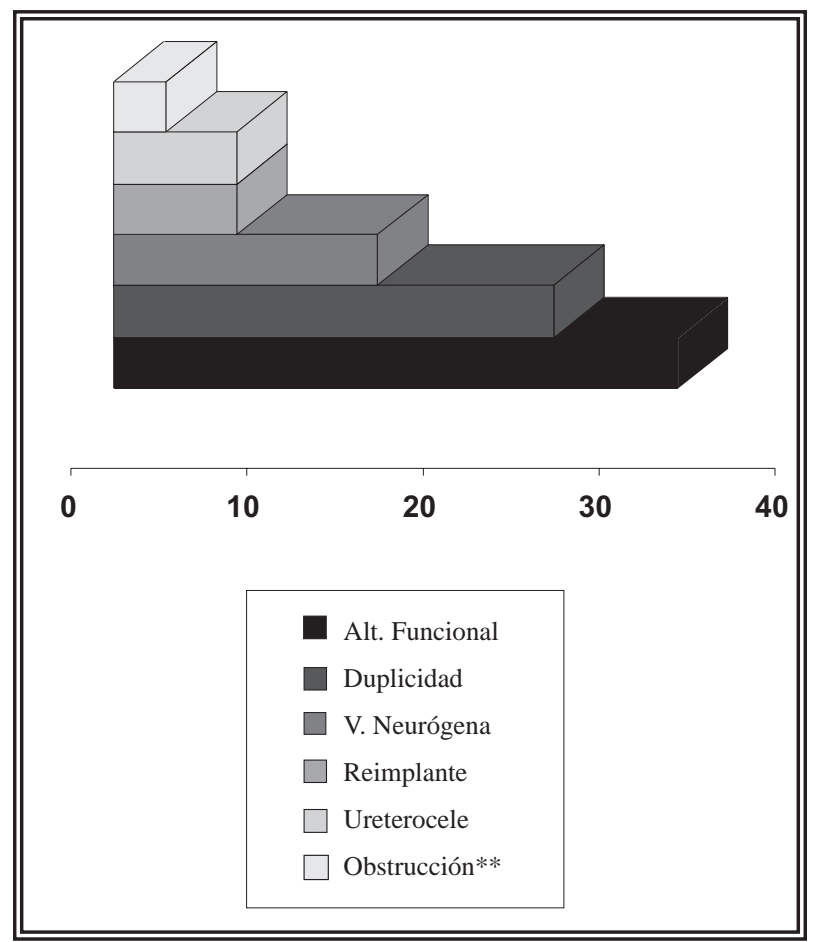

FIGURA 1. Etiología del reflujo vesicoureteral secundario.

Un aspecto técnico merece ser destacado, en inyecciones tras reimplante según Paquin o Politano, realizamos la punción submeática al igual que en reflujos primarios. Cuando se trata de reflujo a uréter implantado según técnica de Cohen, la inyección está dificultada por la situación transversal del uréter, por lo que realizamos primero el cateterismo ureteral para elevar meato y uréter y a continuación inyectamos el polidimetilsiloxano submeático de forma perpendicular al trayecto del uréter, lo más cercano posible al meato. En niños con ureterocele pequeño y RVU a ambos pielones, intentamos una sola inyección submeática al orificio ureteral inferior, el del ureterocele.

La eficacia del tratamiento se definió como la ausencia completa de RVU en la cistografía isotópica realizada a los tres meses tras la punción y posteriormente fueron controlados clinicamente en función de los protocolos de seguimiento de cada una de las patologías de base.

Se estableció una comparación sobre la eficacia del tratamiento entre ambos grupos de pacientes utilizando los estadísticos chi cuadrado, $\mathrm{T}$ de Student y análisis de la varianza. El análisis estadístico se realizó con programa estadístico SPSS versión 12.0 (SPSS, Inc, Chicago, Illinois). El valor de $\mathrm{p}$ significativo fue menor de 0,05 .

\section{RESULTADOS}

De los 124 RVU primarios tratados endoscópicamente el reflujo se corrigió en 96 de ellos $(77,4 \%)$ con un sólo tratamiento y en 19 de los 23 reflujos sometidos a un segundo tratamiento $(82,6 \%)$. En 6 pacientes con reflujo persistente se intentó un tercer tratamiento, resolviéndose uno de ellos (16,6\%). La eficacia global de la técnica en estos pacientes fue el 86,2\% (Tabla 2).

Tabla 2

Eficacia tratamiento endoscópico

\begin{tabular}{|c|c|c|c|c|c|}
\hline Etiologia & $\begin{array}{l}\text { Número } \\
\text { pacientes }\end{array}$ & $\begin{array}{l}\text { 10 tratamiento } \\
\text { \% curación }\end{array}$ & $\begin{array}{l}2^{\circ} \text { tratamiento } \\
\text { \% curación }\end{array}$ & $\begin{array}{l}\text { Eficacia } \\
\text { global \% }\end{array}$ & $\begin{array}{l}\text { Volumen } \\
\text { medio ml }\end{array}$ \\
\hline Reflujo primario & 124 & 71 & 82,6 & 86,2 & 0,72 \\
\hline Alteración funcional* & 32 & 56,3 & 62,5 & 71,87 & 0,72 \\
\hline Duplicidad ureteral & 25 & 72 & 50 & 80 & 0,82 \\
\hline Vejiga neurógena & 15 & 60 & 100 & 80 & 0,90 \\
\hline Reimplante ureteral & 7 & 85,7 & 100 & 100 & 0,84 \\
\hline Ureterocele puncionado & 7 & 57,1 & 100 & 71,4 & 1 \\
\hline Obstrucción orgánica** & 3 & 66,7 & 100 & 100 & 0,84 \\
\hline
\end{tabular}

$(\mathrm{p}=0,712)$

*Incoordinación vesicoesfinteriana o hiperactividad vesical.

**Estenosis de uretra y válvulas de uretra posterior 
De los 32 RVU secundarios a hiperactividad vesical o disinergia vésico-esfinteriana, se resolvieron $18(56,6 \%)$ tras el primer tratamiento. En 8 RVU se intentó un segundo tratamiento corrigiéndose 5 de ellos $(62,5 \%)$ siendo la eficacia global del procedimiento del 71,8\%

Se trataron 25 RVU secundarios a duplicidad ureteral corrigiéndose el reflujo con un sólo tratamiento en 18 (72\%). Tras una segunda inyección se resolvió el RVU en dos de los cuatro pacientes tratados, alcanzando la eficacia global el $80 \%$.

En RVU por disfunción neurogénica la eficacia del primer tratamiento fue del 60\% (curación en 9 de 15 reflujos). De los seis reflujos en los que fracasó el primer tratamiento, 3 se sometieron a una segunda inyección resolviéndose en todos ellos, por lo que la eficacia global en estos RVU fue del $80 \%$.

El tratamiento endoscópico fue eficaz en los 8 pacientes con reflujo persistente tras reimplante ureteral (eficacia del 100\%), precisando uno de ellos 2 tratamientos.

De los 7 reflujos secundarios a ureterocele, 5 se resolvieron por vía endoscópica, 4 ellos con una sola inyección submeática. La eficacia global fue del $71,4 \%$.

Todos los pacientes con reflujo asociado a obstrucción orgánica (tres) se resolvieron con TE, necesitando dos procedimientos uno de ellos.

El análisis univariante no evidenció diferencias significativas al comparar éxitos frente a fracasos según patología de base (Fig 2). En general, la eficacia global del tratamiento endoscópico en reflujos de etiología secundaria fue inferior a la conseguida en el tratamiento del RVU primario $(77,7 \%$ vs. $86,2 \%)$ sin alcanzar estas diferencias la significación estadística $(p=0,226)$.

$\mathrm{El}$ volumen medio inyectado en cada tratamiento endoscópico fue de 0,72 $\mathrm{ml}$ para los pacientes con RVU primario. En los pacientes del grupo II, la cantidad media inyectada varió según la etiología del reflujo, desde los 0,72 $\mathrm{ml}$ de volumen medio en aquellos secundarios a alteración funcional hasta un volumen medio de $1 \mathrm{ml}$ en los reflujos secundarios a ureterocele. Al comparar los volúmenes medios necesarios en cada tratamiento de reflujo primario y en reflujos secundarios según la etiologia, no encontramos diferencias significativas $(\mathrm{p}=0,167)$ en la cantidad de polidimetilsiloxano utilizada (Tabla 3). Tampoco encontramos diferencias significativas al comparar el volumen inyectado y la tasa de resolución del reflujo entre las diferentes etiologías de RVU ( $p=0,361)$.

Se observaron complicaciones en 3 pacientes con reflujo secundario $(4,8 \%)$, todas en el postoperatorio inmediato: un cuadro de hematuria sin repercusión hemodinámica y resolución espontánea, un cuadro de dolor lumbar tipo cólico que cedió con analgesia intravenosa y un tercero requirió tratamiento antibiótico parenteral por fiebre tras la cirugía. La aparición de complicaciones no se asoció a la cantidad de volumen inyectado $(\mathrm{p}=0,704)$, ni influyó en la eficacia del tratamiento $(\mathrm{p}=0,657)$,

Tras un seguimiento medio de 30 meses (1536), únicamente se objetivó recurrencia del RVU en 3 de los 12 pacientes con reflujo secundario a disfunción neurogénica (25\%) y en 1 de los 23 pacientes con RVU por disfunción de vaciado (4,34\%), permaneciendo todos los casos resueltos de RVU secundarios a patología orgánica asintomáticos y sin recidiva

\section{DISCUSIÓN}

La eficacia del (TE) del RVU primario se encuentra validada por muchas series, sin embargo pocos estudios evalúan el tratamiento

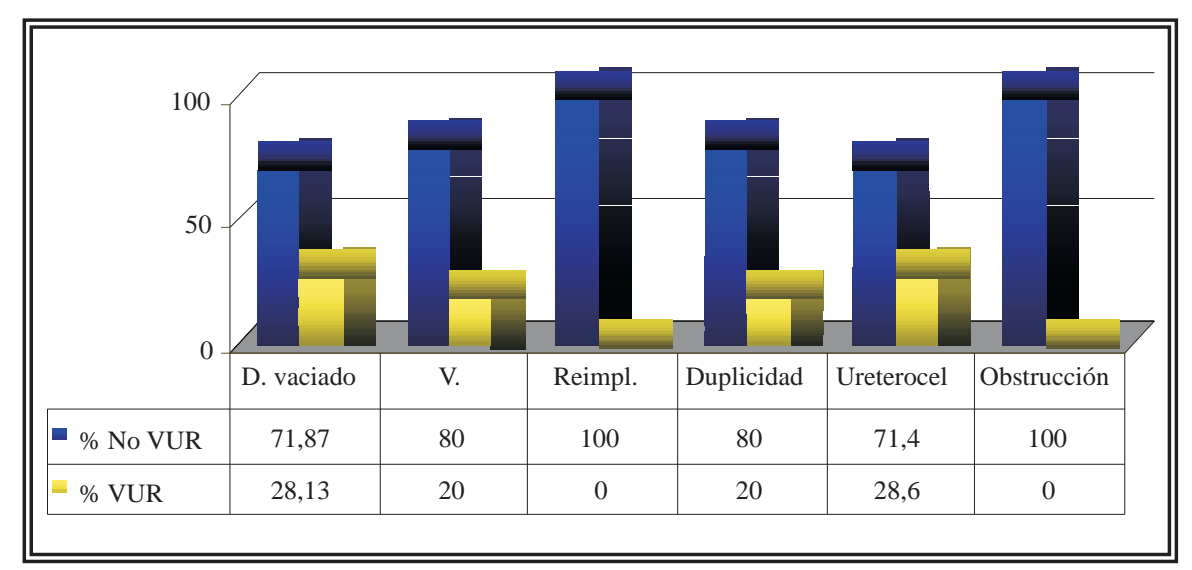

FIGURA 2. Comparación éxitos frente a fracasos tras el tratamiento endoscópico. 
endoscópico en casos complejos de RVU. La morbilidad asociada al procedimiento quirúrgico clásico, el conocimiento de la fisiopatología del tracto urinario y la experiencia adquirida con los agentes inertes, debe estimularnos a progresar en los procedimientos mínimamente invasivos.

Comparando nuestros resultados encontramos que en los pacientes con alteración funcional de base y RVU, obtuvimos una eficacia comparable a la cirugía abierta ${ }^{13}$, con un índice de fracasos del $28 \%$. Sólo un estudio comunica una eficacia cercana al 100\% en una serie de 40 unidades ureterales tratadas con cirugía abierta ${ }^{14}$. Esta diferencia podría explicarse por los diferentes criterios de inclusión, ya que en este estudio sólo trataron a pacientes con alteración en la fase de llenado vesical sin disfunción en el vaciado, en los que cabría esperar peores resultados del (TE) antirreflujo ${ }^{15}$.

Respecto al RVU por duplicidad pieloureteral, el tratamiento clásico consigue unos índices de curación cercanos al $98 \%{ }^{10}$, eficacia superior a la referida por otros autores con tratamiento endoscópico (superior al $73 \%)^{11,16}$ y a la reflejada en nuestra serie (80\%). Sin embargo, las ventajas conocidas del abordaje endoscópico, unido a la posibilidad de tratar el uréter afecto únicamente sin alterar su homolateral sano, frente al tratamiento abierto donde suele ser necesario reimplantar ambos uréteres homolaterales aunque sólo uno presente $\mathrm{RVU}^{17}$, nos haría recomendar este procedimiento inicialmente, dejando la cirugía de reimplante ante fracaso de la técnica endoscópica.

El tratamiento conservador en los pacientes con vejiga neurógena resolvería el $80 \%$ de los reflujos ureterales ${ }^{18}$. Según las series, el reimplante ureteral curaría hasta el 99\% de los reflujos en los que fracasa el tratamiento conservador $^{19}$, lo que unido al índice de fracasos del tratamiento endoscópico con colágeno, llevó a algunos autores a desestimar la utilización de agentes inertes en esta patología ${ }^{9}$. La utilización de nuevos agentes inertes como el teflón mejoraron los resultados del tratamiento endoscópico ${ }^{20}$, similares a los obtenidos en nuestra serie con polidimetilsiloxano ( $80 \%$ de eficacia global), por lo que actualmente debería ser considerado como tratamiento inicial en esta patología por las ven- tajas ya comentadas del abordaje endoscópico y por la necesidad de minimizar las agresiones quirúrgicas en pacientes subsidiarios de más cirugias futuras.

En pacientes con RVU persistente tras reimplante ureteral, el (TE) es técnicamente más complejo y precisa en muchas ocasiones de cateterismo ureteral que también suele ser dificultoso. Sin embargo, si consideramos que la ureterolisis tras reimplante es muy laboriosa y que el tratamiento endoscópico curó el RVU en todos los pacientes tratados en nuestra serie, se justificaría la vía endoscópica para el tratamiento del reflujo ante fracaso del reimplante ureteral, si bien es cierto que este tipo de procedimientos son cada vez más infrecuentes.

Cuando tratamos el reflujo residual del ureterocele puncionado o el reflujo al uréter del pielón inferior al del ureterocele por vía endoscópica, encontramos diferencias en los resultados al compararlos con la literatura (eficacia del 71,4\% vs. $60 \%)^{11}$. Una posible explicación podría hallarse en la técnica de punción utilizada y en la selección rigurosa de los casos, tratando únicamente ureteroceles de pequeño tamaño, pues difícilmente el RVU en grandes ureteroceles puede ser resuelto de forma endoscópica

En los reflujos secundarios a válvulas de uretra posterior o estenosis de uretra, nuestros resultados fueron excelentes en los pacientes seleccionados, al igual que otros autores que también refieren un elevado índice de curación ${ }^{11}{ }^{21}$ con este abordaje endoscópico. Por ello, aunque las series publicadas son cortas, debería ser considerada una alternativa válida de tratamiento.

Hemos observado que la eficacia del (TE) es mantenida a lo largo del tiempo con resultados comparables a los obtenidos en el tratamiento endoscópico del RVU primario ${ }^{2,5,10}$. Sin embargo la recurrencia del reflujo en algunos pacientes con alteración funcional vesical obliga al seguimiento a largo plazo en este tipo de patologías, con patrones urodinámicos variables a lo largo del tiempo.

\section{CONCLUSIONES}

El tratamiento endoscópico de RVU de etiología secundaria es una técnica mínimamente invasiva, presenta escasa morbilidad y es eficaz en el RVU de etiología secundaria en pacientes seleccionados. 
El riesgo de recurrencia a largo plazo es mayor en el reflujo secundario a alteraciones funcionales (vejiga neurógena e inestabilidad funcional), por lo que el seguimiento debería establecerse según la patología de base.

\section{REFERENCIAS}

1. Misra D, Potts SR, Brown S, Boston VE. Endoscopic treatment of vesico-ureteric reflux in neurogenic bladder-8 years' experience. J Pediatr Surg 1996;31(9):1262-1264.

2. Chertin B, Colhoun E, Velayudham M, Puri P. Endoscopic treatment of vesicoureteral reflux: 11 to 17 years of followup. J Urol 2002;167(3):1443-1445; discussion 14451446.

3. Matouschek E. Sobre un nuevo concepto para el tratamiento del reflujo vesicoureteral. Arch. Esp. Urol 1981;34 (5):385-388.

4. O'Donnell B, PURI P. Treatment of vesicou-ureteric reflux by endoscopic injection of Teflon. Brit Med J 1984;289:7-9

5. Capozza N, Lais A, Nappo S, Caione P. The role of endoscopic treatment of vesicoureteral reflux: a 17-year experience. J Urol 2004;172 (4 Pt 2):1626-8; discussion 1629

6. Puri P, Granata C. Multicenter survey of endoscopic treatment of vesicoureteral reflux using polytetrafluoroethylene. J Urol 1998;160:(3 Pt2):1007-1011; discussion 1038.

7. Serrano-Durba A, Serrano AJ, Magdalena JR, Martin JD, Soria E, Dominguez C, et al. The use of neural networks for predicting the result of endoscopic treatment for vesico-ureteric reflux. BJU Int 2004;94(1):120-122.

8. Serrano-Durba A, Estornell F, Dominguez C, Martínez M, Garcia-Ibarra F. Eficacia del tratamiento endoscópico en el reflujo vesicoureteral primario en la infancia con polidimetilsiloxano. Urol Integr Invest 2002;7:490-493.

9. Haferkamp A, Mohring K, Staehler G, Gerner HJ, Dorsam J. Long-term efficacy of subureteral collagen injection for endoscopic treatment of vesicoureteral reflux in neurogenic bladder cases. J Urol 2000;163(1):274-277.

10. Lackgren G, Wahlin N, Skoldenberg E, Neveus T, Stenberg A. Endoscopic treatment of vesicoureteral reflux with dextranomer/hyaluronic acid copolymer is effective in either double ureters or a small kidney. J Urol 2003;170(4 Pt2): 1551-1555; discussion 1555.
11. Perez-Brayfield M, Kirsch AJ, Hensle TW, Koyle MA, Furness P, Scherz HC. Endoscopic treatment with dextranomer/hyaluronic acid for complex cases of vesicoureteral reflux. J Urol 2004;172(4 Pt2):1614-1616.

12. O’Donnell B, Puri P. Treatment of vesicoureteric reflux by endoscopic injection of Teflon1984. J Urol. 2002;167(4): 1808-1809

13. Nasrallah PF, Simon JW. Reflux and voiding abnormalities in children. Urology 1984;24(3):243-245.

14. Barroso U, Jr., Jednak R, Barthold JS, Gonzalez R. Outcome of ureteral reimplantation in children with the urge syndrome. J Urol 2001;166(3):1031-1035.

15. Noe HN. The role of dysfunctional voiding in failure or complication of ureteral reimplantation for primary reflux. J Urol 1985; 134: 1172-1175.

16. Aboutaleb H, Bolduc S, Khoury AE, Upadhyay J, Bagli DJ, Farhat W. Polydimethylsiloxane injection versus open surgery for the treatment of vesicoureteral reflux in complete duplex systems. J Urol 2003;170(4 Pt2):1563-1565.

17. Ortenberg J. Endoscopic treatment of vesicoureteral reflux in children. Urol Clin North Am 1998;25:151-156.

18. Agarwal SK, Khoury AE, Abramson RP, Churchill BM, Argiropoulos G, McLorie GA. Outcome analysis of vesicoureteral reflux in children with myelodysplasia. J Urol 1997; 157(3):980-982.

19. Engel JD, Palmer LS, Cheng EY, Kaplan WE. Surgical versus endoscopic correction of vesicoureteral reflux in children with neurogenic bladder dysfunction. J Urol 1997; 157(6):2291-2294.

20. Granata C, Buffa P, Di Rovasenda E, Mattioli G, Scarsi PL, Podesta E, et al. Treatment of vesico-ureteric reflux in children with neuropathic bladder: a comparison of surgical and endoscopic correction. J Pediatr Surg 1999;34(12): 1836-1838.

21. Puri P, Kumar R. Endoscopic correction of veesicoureteral reflux secondary to posterior urethral valves. J Urol. 2001;166:1887-1892.

Dr. A. Serrano Durbá

E-mail: serrano_agu@gva.es

(Trabajo recibido el 23 de noviembre 2006) 